\title{
Exogenous neuregulin-1 attenuates STZ-induced diabetic peripheral neuropathic pain in rats ${ }^{1}$
}

Fang Zhou', Zhongyuan Xia", Kang Liu"', Qin Zhou'"'

'Master, Department of Anesthesia, Renmin Hospital, Wuhan University, China. Conception and design of the study, acquisition and interpretation of data, manuscript writing.

"PhD, Full Professor, Department of Anesthesia, Renmin Hospital, Wuhan University, China. Design and supervised all phases of the study, critical revision.

'"Master, Department of Anesthesia, Renmin Hospital, Wuhan University, China. Acquisition and interpretation of data, critical revision.

\section{Abstract}

Purpose: To investigate whether modulating NRG1 could attenuate diabetic neuropathic pain and analyze the underlying mechanism.

Methods: Male SD rats were randomly divided into control group, diabetic group, NRG1 intervention group. After STZ-induced 2 weeks, NRG1 intervention daily for consecutive 7 days. 4 weeks after NRG1 intervention, both the mechanical withdrawal threshold and the morphological changes of the dorsal root ganglion and sural nerve were observed. Meanwhile, the expression of NGF, IL-1 $\beta$, TNF- $\alpha$ in spinal cord were determined.

Results: Compared with the diabetic group, NRG1 treatment improved the mechanical withdrawal threshold in diabetic rats, pathological changes of dorsal root ganglion and sural nerve were alleviated by NRG1 treatment with electron microscopy imagine. Moreover, compared with the control group, the expression of NGF was significantly decreased and the production of IL-1 $\beta$, TNF- $\alpha$ were markedly induced in diabetic group. Furthermore, NRG1 treatment could normalized the above effect as compared to diabetic group.

Conclusion: NRG1 exerted positive effects on the behavioral and pathological changes of rats with STZ-induced diabetic neuropathic pain, the underlying mechanism might be related to the promotion of NGF excretion and the inhibition of inflammatory cytokines excretion.

Key words: Neuregulin-1. Diabetes Mellitus. Pain. Nerve Growth Factor. Cytokines. Rats. 


\section{- Introduction}

Diabetic peripheral neuropathic pain (DNP) is a common microvascular complication of diabetes mellitus, more than $50 \%$ of diabetic patients are suffered from this painful molestation $^{1}$, it can severely affect patients' daily lives. Spontaneous pain, allodynia (pain perception in response to normally non-painful stimuli) and hyperalgesia (exaggerated pain sensations to normally painful stimuli) are the main characters of DNP. There are several acceptable pathogenetic factors which can induce diabetic neuropathy pain, including polyalcohol pathway, non-enzymatic protein glycosylation, abnormal lipid metabolism, the deficiency of neurotrophic factor, oxygen-free radical damage and so on. Clinically, this painful molestation is extremely difficult to prevent and treat.

Neuregulin-1(NRG1) is one of the component of Neuregulins (NRGs), which belong to the large epidermal growth factors (EGF) family of proteins. Till now, NRG1 is the most extensively characterized of the NRGs family, which has a key role in neural and cardiac development. NRG1 can not only modulate synaptic plasticity, but also stimulate the proliferation, survival and motility of a number of different cell types through binding to ErbB tyrosine kinase $\mathrm{e}^{2-4}$. It has also shown that NRG1 is required for the development of the peripheral nervous system. As we known, Schwann cells (SCs) are critical for myelin sheath thickness and intermodal distance of nerve conduction velocity, they are also necessary for the recovery from nerve injury ${ }^{5}$. NRG1 can regulate multiple aspects of SC differentiation including the survival of SC precursors, proliferation, motility, axon ensheathment and myelination ${ }^{6,7}$, it can also promote neuronal regeneration and differentiation in the peripheral nervous system by expressing neurotrophic factors including NGF. Recent studies have confirmed NRG1 is required for remyelination and regeneration following nerve injury, and increasing the expression of NRG1 and ErbB4 could relieve neuropathic pain induced by spinal cord injury ${ }^{8}$. Other report showed that both single NRG1 injection and chronic NRG1 treatment could improve glucose tolerance ${ }^{9}$. Though a substantial body of evidence suggests that NRG1 plays a key role in the recovery of nerve injury, but whether modulating NRG1 function can prevent and cure DNP remains unclear.

As mentioned before, previous studies have shown beneficial effects of NRG1 such as impaired remyelination, peripheral regeneration, the survival of SCs, reduction of inflammation. So exogenous NRG1 may be useful in the treatment of the damage inevitably followed by diabetic neuropathy. To address this question, in the present study we investigated the behavioral and pathological changes in STZ-induced rats. Moreover, in order to clarify the underlying mechanism, we evaluated the impact of NRG1 on the NGF level, TNF- $\alpha$ and IL-1 $\beta$ levels in spinal cord of diabetic rats.

\section{- Methods}

All procedures of this experiment were approved by the ethics committee of the Renmin Hospital of Wuhan University, Wuhan, China. All experimental procedures complied with the Guide for the Care and Use of Laboratory Animals.

Male Sprague-Dawley rats $(n=35)$, body weights 200-220g, were housed in separate cages at constant temperature $\left(20-22^{\circ} \mathrm{C}\right)$ and humidity (50\%-67\%) under a 12-hour light/dark cycle in specific pathogen free animal laboratory of Renmin Hospital of Wuhan university.

\section{STZ-induced diabetes}

The methods used in this model were described previously ${ }^{10}$. The rats were randomly divided into two groups, the STZ-induced group $(n=25)$ and the control group $(n=10)$. Rats in the STZ-induced group were fed on high fat and 
high sugar diets, while rats in the control group were fed with the routine diets. After one week of acclimatization, the STZ-induced rat model was established by injecting STZ $(55 \mathrm{mg} / \mathrm{kg}$ body weight, Sigma-Aldrich, St Louis, MO, USA) intraperitoneally. Seventy two hours later, the plasma glucose levels were determined by the glucose oxidase method using the one touch ultra blood glucose meter, and rats with the blood glucose more than $16.7 \mathrm{mmol} / \mathrm{L}$ were considered to be successfully induced for diabetes. After the successful construction of the rat diabetic models, the rats in the STZ-induced group were divided into diabetic group $(n=11)$ and NRG1 intervention group $(n=10)$.

\section{NRG1 intervention}

Two weeks after the injection of STZ, twenty-one diabetic rats were randomly assigned to receive either $10 \mu \mathrm{g} \bullet \mathrm{kg}^{-1} \bullet \mathrm{d}^{-1} \mathrm{rhNRG1}$ (Sino Biological Inc. Lot: LCO8SE2815, $n=10$ ) intravenous injection for 7 consecutive days, or vehicle (saline, $n=11$ ), while the ten control rats received no treatment. The dose of rh-NRG1 was chosen based on a previous study ${ }^{11}$.

\section{Behavioral Assessment of mechanical sensitivity}

Von Frey test was used to determine the mechanical sensitivity threshold for nociception. Each rat was placed in a chamber with a mesh metal floor, which permitted rat freedom of movement while allowing investigators access to their paws. Then, von Frey hairs, ranging from 0.25 to 15 grams, were applied 5 times, and was scored positive when a minimum of 3 paw flicks (the animal's reflex withdrawal response) were observed, as described previously. The control group served as the reference group.

\section{Western bolts}

Seven weeks after injection of STZ, the rats were sacrificed. They were anesthetized with
$1 \%$ pentobarbital sodium $(50 \mathrm{mg} / \mathrm{kg}$ body weight, i.p.), then the lumbar enlargement of spinal cord and the $L^{4,5}$ dorsal root ganglions (DRGs) were rapidly removed and quickly frozen in liquid nitrogen. Tissue samples were homogenized in a phosphate buffer with protease inhibitor cocktail, the lysate were spun at $15.000 \mathrm{rpm}$ at $4^{\circ} \mathrm{C}$ for 15 minutes and the protein concentration of supernatant was determined using a BCA protein assay kit. Proteins $(50 \mu \mathrm{g} / \mathrm{sample})$ were separated using 8\% SDS-PAGE, and transferred to nitrocellulose membranes. Membranes were then blocked in 10\% skimmed milk for 1 hour at room temperature and washed in PBS-T for three times at 5 minutes each. Membranes were incubated with primary antibody, rabbit antiNRG-1(1:200), rabbit anti-NGF (1:600), rabbit anti-IL-1 $\beta$ (1:200), rabbit anti-TNF- $\alpha$ (1:200) overnight at $4^{\circ} \mathrm{C}$. Appropriate HRP-conjugated secondary antibodies were applied for 1 hour. After several PBS-T washes as described above membranes were revealed using ECL plus reagent for detection by autoradiography.

\section{Electron microscopy}

The DRGs and sural nerve were removed and fixed overnight at $4^{\circ} \mathrm{C}$ in the $2 \%$ glutaraldehyde for 24 hours, then washed in $0.1 \mathrm{M}$ phosphate buffer, osmicated, dehydrated and embedded in epoxy resin. After double staining with uranyl acetate and lead nitrate, morphological changes of the DRGs and sural nerves were observed by transmission electron microscopy (HT7700, Hitachi, Japan). Images were taken by imaging systems (Moticam2306, Motic instruments Inc., Canada).

\section{Statistical analysis}

The SPSS 19.0 software was used for statistical analysis. All results were presented as the mean \pm SD. Student's t-test was performed to determine the differences of the mechanical withdrawal threshold. Other datas were used ANOVA for random measures and LSD-t for multiple 
comparison, and $\mathrm{P}<0.05$ were considered as statistically significant.

\section{Results}

Development of hyperglycemia in STZ-induced rats

In our study, most of the rats $(84 \%, 21$ out of 25) developed hyperglycemia following STZ injection, this hyperglycemia started on the third day after STZ injection and persisted during the entire experimental period.

\section{NRG1 treatment improved the mechanical withdrawal threshold in diabetic rats}

To investigate the effect of NRG1 on diabetic neuropathy pain, we performed experiments two weeks after STZ injection, in which NRG1 was i.v. administered to rats for 7days. As shown in Figure 1, administration of NRG1 significantly attenuated the development of mechanical allodynia in diabetic rats. Quantitative analysis showed that NRG1 increased the mechanical pain threshold with the extension of time.

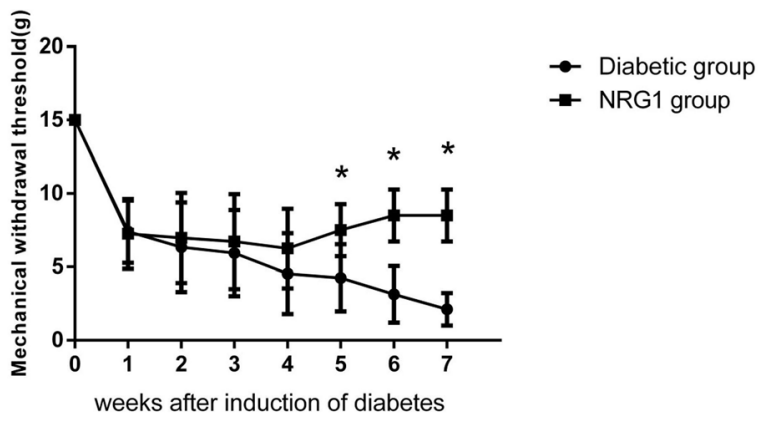

Figure 1 - NRG1 alleviated mechanical hypersensitivity in diabetic rats. The mechanical withdrawal threshold recorded in the NRG1 intervention group revealed a significant change compared with the diabetic group from the fifth week after induction of diabetes $\left({ }^{*}, \mathrm{P}<0.05\right.$ versus diabetic group, $n=10$ ). Data was presented as mean $\pm S D$.

\section{NRG1 treatment increased NGF level in spinal cord}

NRG1 can modulate Schwann cell proliferation following injury, and one of the important functions of Schwann cells is to secrete NGF. Previous evidences showed that NGF played a significant role in the pathogenesis of diabetic polyneuropathy, and itself had been considered an option for the treatment of diabetic peripheral pain ${ }^{12}$. In our study, we confirmed the expression of NRG1 and NGF in spinal cord of rats. As shown in Figure 2, we found that compared to control group, both in the NRG1 intervention group and the diabetic group, the expressions of NRG1 and NGF were significantly decreased $(P<0.05)$. Although compared to the diabetic group, there was an increase of NRG1 expression in the NRG1 intervention group, it did not reach significance, while the expression of NGF increased in the NRG1 intervention group, and there was statistical significance between the two groups $(P<0.05)$.

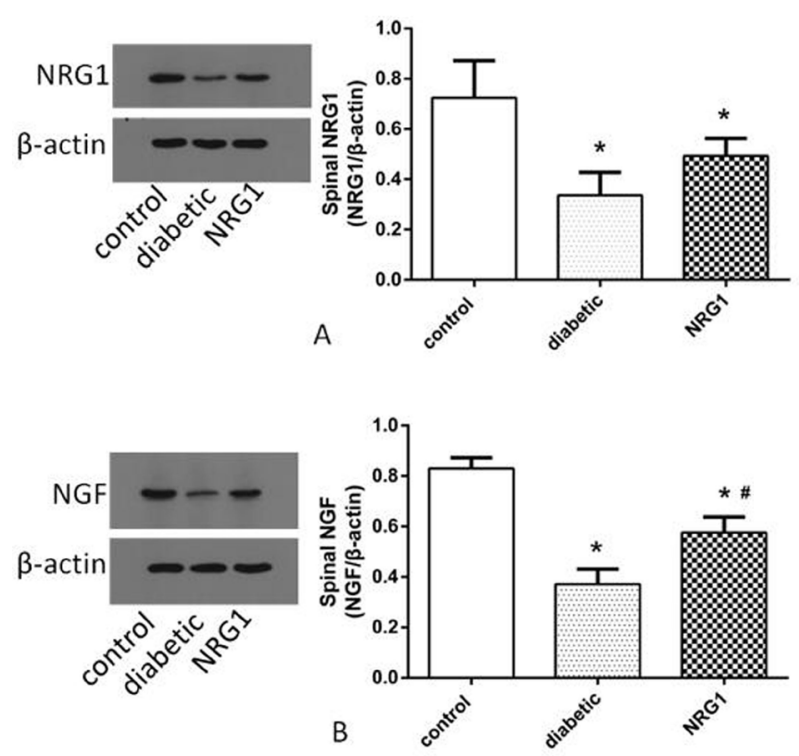

Figure 2 - The expression of NRG1, NGF in spinal cord. A representative western blot and the grey value analysis of protein expression in spinal cord from different experimental groups. Data were expressed as mean $\pm \mathrm{SD}$. ${ }^{*} \mathrm{P}<0.05$ vs control group, $\# \mathrm{P}<0.05$ vs diabetic group. 
NRG1 treatment reduced TNF- $\alpha$ and IL-1B levels in spinal cord

Previous studies have indicated that the over-expression of TNF- $\alpha$ and IL-1 $\beta$ could lead to pain hypersensitivity ${ }^{13,14}$, and NRG1 played an important role in the molecular mechanisms of inflammatory regulated disorder ${ }^{15}$. In our study, we confirmed the expression of TNF- $\alpha$ and IL-1 $\beta$ in spinal cord by western blot analysis. As shown in Figure 3, compared with the control group, the protein expression of TNF- $\alpha$ and IL- $\beta$ increased in both diabetic group and NRG1 intervention group $(P<0.05)$, while compared with the diabetic group, the protein expression of TNF- $\alpha$ and IL- $\beta$ in NRG1 intervention group decreased, and there were significant differences between the two groups $(P<0.05)$.

Effects of NRG1 on the histological features of
DRGs and sural nerve in diabetic rats
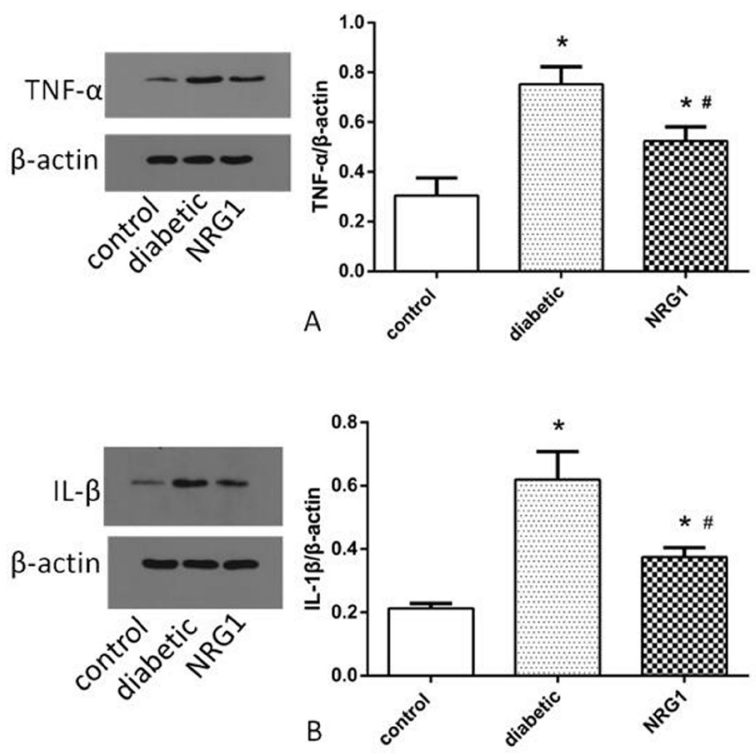

Figure 3 - The expression of TNF- $\alpha$, IL- $\beta$ in spinal cord. Data were expressed as mean \pm SD. ${ }^{*} \mathrm{P}<0.05$ vs control group, $\# \mathrm{P}<0.05$ vs diabetic group.

As shown in Figures 4 and 5, we clearly found that NRG1 could alleviate pathological injury of DRGs and sural nerve.
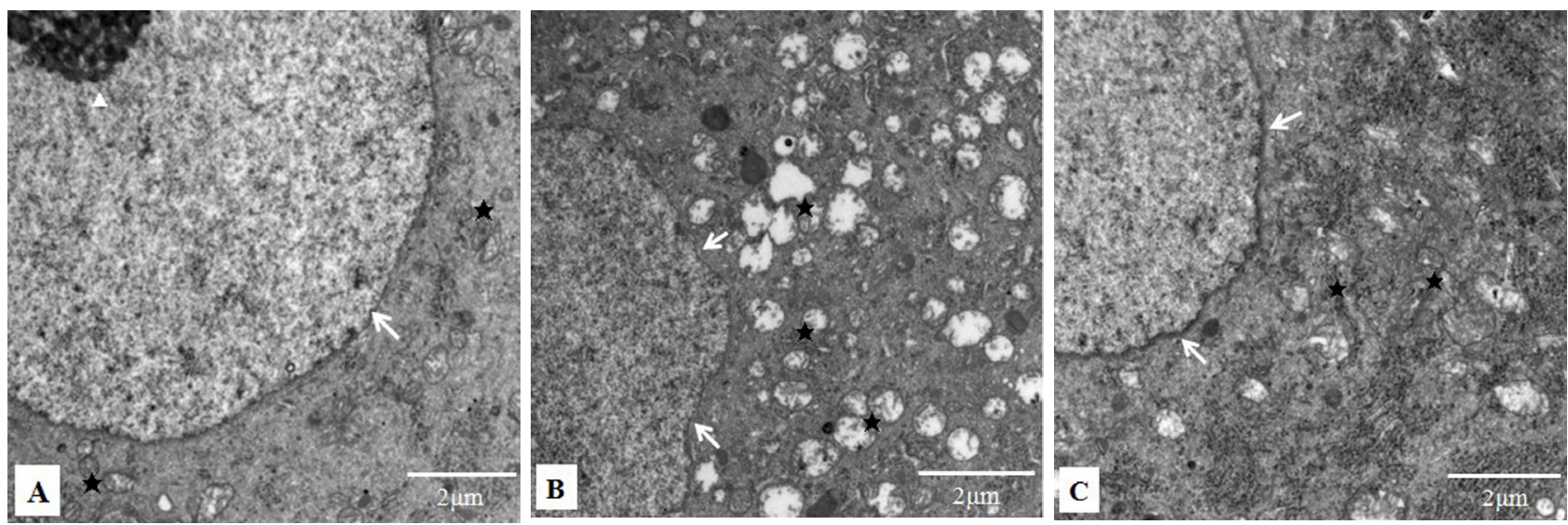

Figure 4 - Transmission electron micrographs showing the ultrastructure of dorsal root ganglion $(\times 2500)$. A. Control group: the nucleus $(\boldsymbol{\Delta})$ was complete, the nuclear membrane was generally smooth (arrow), there was no significant swelling of mitochondrial( $\star$ ). B. Diabetic group: The nuclear membrane was shrink (arrows), the nucleolus was not clear, there were large vacuolar degeneration in cytoplasm, there was significant swelling of rough endoplasmic reticulum $(\star)$. C. NRG1 intervention group: The nuclear membrane was basically integral (arrows), slight vesiculation was observed in the nucleus and cytoplasm ( $\star$ ). 

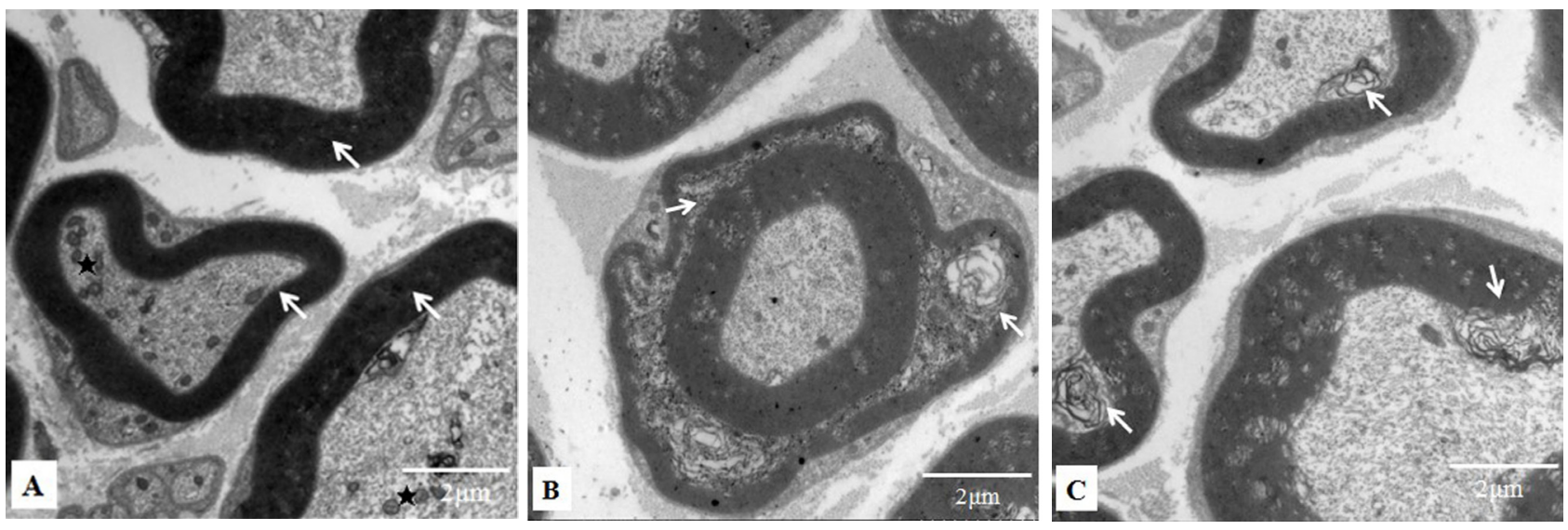

Figure 5 - Transmission electron micrographs showing the ultrastructure of sural nerve $(\times 2500)$. A. Control group: intact myelinated axon, the myelin sheath was well-distributed (arrows), mitochondria with normal morphology could be seen in the axon ( $\star$ ). B. Diabetic group: serious demyelination, the myelin sheath was arranged in disorder, broken and swollen were observed (arrows). C. NRG1 intervention group: moderate segmental demylination, the myelin sheath was arranged disorder in local areas, and a small amount of vacuolization could be seen (arrows).

\section{- Discussion}

By utilizing a combination of behavioral test and electron microscopy, the present study demonstrated that exogenous NRG1 could alleviate STZ-induced DNP in rats, it could reduce the necrosis of the DRG and relieve the demyelination of sural nerve. This study provides direct evidence for a crucial role of NRG1 in function and morphology of diabetic neuropathy, and the protective effects may be attributed to a result of increasing excretion of NGF and inhibiting release of inflammatory factors.

\section{Exogenous NRG1 can inhibit diabetic neuropathic pain}

In our study, we found that when given exogenous NRG1, the pain threshold of rats in the NRG1 intervention group was raised. As we known, the pathologic mechanisms of DNP are associated with microcirculatory disturbance and metabolism disorders. The microcirculatory disturbance reduces peripheral nerve perfusion and causes endoneurial hypoxia, the hyperglycemia-induced polyol pathway as well as enhanced oxidative stress are possibly exerted by direct nerve tissue damage or mediated by endothelial injury or vascular dysfunction. Both of them finally lead to demyelination of nerve fiber, degeneration of axon and change of neurotransmitters, and all of these changes can lead to diabetic neuropathy. In, present study, apparently demyelination of sural nerve and cell degeneration of the DRGs were emerged in the diabetic group, these were consistent with a model of neuropathic pain.

NRG1 is a family of growth and differentiation factors involved in various functions of both peripheral and central nervous system including the regenerative processes that underlie regeneration of damaged peripheral nerves ${ }^{16}$. It is now known to be a key axon-derived factor in determining axon ensheathment and peripheral myelin thickness ${ }^{8}$, Fricker et al. ${ }^{17}$ observed that axons lacking NRG1 had significantly thinner myelin sheaths and $38.5 \pm 3.3 \%$ had completely failed to elaborate a myelin sheath despite being ensheathed by $\mathrm{SCs}$ at 2 months following sciatic nerve crush. 
There also a number of studies suggested that NRG1 expression levels were dramatically reduced after injury, both spontaneous painlike behaviors and mechanical hypersensitivity could be reversed by application of exogenous $N_{R G}{ }^{18,18-20}$. Our results were consistent with these previous studies, compared with the control group the level of NRG1 in the enlargement of spinal cord was apparently decreased in diabetic rats, the necrosis of DRGs were reduced and the demyelination of sural nerve was apparently improved in the NRG1 intervention group, although there was no significant difference of the expression of NRG1 between diabetic group and NRG1 intervention group. These evidences from the other side showed that NRG1 alleviated DNP might not by direct influence but through other signal pathways, which would be mentioned below.

The preventive efficacy of exogenous NRG1 in DNP might be related to NGF secretion The success of peripheral nerve regeneration is critically depended on the local environment of the distal nerve stump after injury. Nerve growth factor (NGF), normally synthesized in neuronal target tissues and retrogradely transported to the neuronal cell body to exert its trophic functions, is essential for the survival and maintenance of sympathetic and sensory nerves ${ }^{21,22}$. It can stimulate the growth of axon, maintain the diameter of axon, prevent the death of adult neurons after injury, regulate the plasticity of synaptic and the transmission of neurotransmitter. Recent reports have suggested that reduced levels of NGF plays a significant role in the pathogenesis of diabetic neuropathy. Retrograde axonal transport of NGF is impaired in animals with DM, and its transcription in neuronal target tissues is reduced. Pathological conditions that alter NGF expression may result in neuronal dysfunction and death ${ }^{12}$. A previous study showed that the concentration of NGF in the sciatic nerves of STZ-induced diabetic rats decreased 3 weeks after STZ injection ${ }^{23}$. In our study, the level of NGF in the spinal cord were markedly reduced in diabetic group, these were consistent with the previous studies, it indicated a loss of neural integrity and an increased rate of nerve cell apoptosis. And this indication was confirmed by electron microscopy of the DRGs. As mentioned before, there has a close relationship between NRG1 and SCs. NRG1 secreted by peripheral axons has previously been identified as an essential factor for SCS proliferation and migration ${ }^{24}$. SCs play a critical role in promoting neuronal regeneration and differentiation in the peripheral nervous system by expressing neurotrophic factors, including NGF. One of the important functions of SCs is to secrete NGF and high concentrations of glucose can lead to the reduction of NGF production in $\mathrm{SCs}^{25}$. The present study confirmed the previous results. After given exogenous NRG1, the NGF level apparently increased. So we concluded that NRG1 might ameliorated DNP through the secretion of NGF, and this conclusion depends on further experiments to confirm.

\section{NRG1 can inhibit the release of inflammatory factors}

A substantial body of evidence suggests that oxidative stress plays a major role among the mechanisms of diabetic neuropathy. The expression of pro-inflammatory cytokines, such as IL-1 $\beta$ and TNF- $\alpha$ in the dorsal horn of the spinal cord is thought to contribute to the pathogenesis of diabetic neuropathy ${ }^{26,27}$. Long term hyperglycemia can damage the vascular barrier of the nerves. Myelin protein glycosylation alters its antigenicity, promotes the phagocytic function of mononuclear macrophages from blood circulation and tissue as well as glial cells from nervous system. Then the activated immune cells secrete cytokines such as IL-1 $\beta$ and TNF- $\alpha^{28}$. They target voltagegated ion channels in cell membrane such as $\mathrm{K}^{+}, \mathrm{Ca}^{2+}$ channels, rapidly increase the neuronal excitability, and lead to a sustained increase in ion channels' conductivity, all of these results in the occurrence of pain. Previous preclinical studies have demonstrated an association 
between elevated IL-1 $\beta$, TNF- $\alpha$ and altered pain behavior. We also found that both IL-1 $\beta$ and TNF- $\alpha$ were lower in the NRG1intervention group than the diabetic group. Recent evidence suggested that NRG1might play a role in regulation of inflammation and immune system response. Ketan Marballi's study firstly reported statistical association between a mutation in NRG1 and immune system dysregulation, which contributed to the understanding of the role of NRG1 in the pathogenesis of schizophrenia and other disorders in which inflammation played an important role ${ }^{29}$. So we hypothesized that NRG1 could inhibit the release of inflammatory cytokines in diabetic rats.

\section{Conclusions}

Neuregulin-1 could alleviate diabetic neuropathic pain via its regulation of NGF and anti-inflammatory.

\section{- References}

1. Tesfaye S, Selvarajah D. Advances in the epidemiology, pathogenesis and management of diabetic peripheral neuropathy. Diabetes Metab Res Rev. 2012 Feb;28 Suppl 1:8-14. doi: 10.1002/ dmrr.2239.

2. Li B, Xiao J, Li Y, Zhang J, Zeng M. Gene transfer of human neuregulin-1 attenuates ventricular remodeling in diabetic cardiomyopathy rats. Exp Ther Med. 2013 Nov;6(5):1105-12. PMID: 24223630.

3. Min SS, An J, Lee JH, Seol GH, Im JH, Kim HS, Baik TK, Woo RS. Neuregulin-1 prevents amyloid beta-induced impairment of longterm potentiation in hippocampal slices via ErbB4. Neurosci Lett. 2011 Nov;505(1):6-9. PMID: 21787838.

4. Engel M, Snikeris P, Jenner A, KarlT, Huang XF, FrankE. Neuregulin1PreventsPhencyclidineInduced Behavioral Impairments and Disruptions to GABAergic Signaling in Mice. Int J Neuropsychopharmacol. 2015 May;18(7):pyu114. PMID: 26478928.

5. Zou DW, Gao YB, Zhu ZY, Zhou H,
ZhangTJ, Li BM, Wang JY, Li MZ, Zhang N. Traditional chinese medicine tang-luoning ameliorates sciatic nerve injuries in streptozotocin-induced diabetic rats. Evid Based Complement Alternat Med. 2013;2013:989670. PMID: 24288572.

6. Cornejo M, Nambi D, Walheim C, Somerville M, Walker J, Kim L, Ollison L, Diamante G,Vyawahare S, de Bellard ME. Effect of NRG1, GDNF, EGF and NGF in the migration of a Schwann cell precursor line. Neurochem Res. 2010 Oct;35(10):1643-51. PMID: 20623378.

7. Heermann S, Schmucker J, Hinz U, Rickmann M, Unterbarnscheidt T, Schwab $\mathrm{MH}$, Krieglstein K. Neuregulin 1 type III/ ErbB signaling is crucial for Schwann cell colonization of sympathetic axons. Plos One. 2011;6(12):e28692. doi: 10.1371/ journal.pone. 0028692.

8. Tao F, Li Q, Liu S, Wu H, Skinner J, Hurtado A, Belegu V, Furmanski O, Yang Y, McDonald JW, Johns RA.. Role of neuregulin-1/ErbB signaling in stem cell therapy for spinal cord injury-induced chronic neuropathic pain. Stem Cells. 2013 Jan;31(1):83-91. doi: 10.1002/stem.1258

9. Ennequin G, Boisseau N, Caillaud K, Chavanelle V, Etienne M, Li X, Sirvent P. Neuregulin 1 Improves Glucose Tolerance in db/db Mice. Plos One. 2015 Jul31;10(7):e130568. doi: 10.1371/journal. pone. 0130568.

10. Tong $M$, Tuk $B$, Shang $P$, Hekking IM, Fijneman EM, Guijt $M$, Hovius $S E$, van Neck JW. Diabetes-impaired wound healing is improved by matrix therapy with heparan sulfate glycosaminoglycan mimetic OTR4120 in rats. Diabetes. 2012 Oct;61(10):2633-41. PMID: 22721969.

11. Liu X, Gu X, Li Z, Li X, Li H, Chang J, Chen P, Jin J, Xi B, Chen D, Lai D, Graham RM, Zhou M. Neuregulin-1/erbB-activation improves cardiac function and survival in models of ischemic, dilated, and viral cardiomyopathy. J Am Coll Cardiol. 2006 Oct;48(7):1438-47. doi: 10.1016/j.jacc.2006.05.057.

12. Kang TH, Moon E, Hong BN, Choi SZ, Son M, Park JH, Kim SY. Diosgenin from Dioscorea 
nipponica ameliorates diabetic neuropathy by inducing nerve growth factor. Biol Pharm Bull. 2011;34(9):1493-8. PMID: 21881239.

13. Ren K, Torres R. Role of interleukin-1beta during pain and inflammation. Brain Res Rev. 2009 Apr;60(1):57-64. PMID: 19166877.

14. Kawasaki Y, Zhang L, Cheng JK, Ji RR. Cytokine mechanisms of central sensitization: distinct and overlapping role of interleukin-1beta, interleukin-6, and tumor necrosis factor-alpha in regulating synaptic and neuronal activity in the superficial spinal cord. J Neurosci. 2008 May;28(20):5189-94. doi: 10.1523/ JNEUROSCI.3338-07.2008.

15. Marballi K, Quinones MP, Jimenez F, Escamilla MA, Raventos $H$, Soto-Bernardini MC, Ahuja SS, Walss-Bass C. In vivo and in vitro genetic evidence of involvement of neuregulin 1 in immune system dysregulation. J Mol Med (Berl). 2010 Nov;88(11):1133-41. PMID: 20625696.

16. Fricker FR, Bennett DL. The role of neuregulin-1 in the response to nerve injury. Future Neurol. 2011 Nov;6(6):80922. PMID: 22121335.

17. Fricker FR, Lago N, Balarajah S, Tsantoulas C, Tanna S, Zhu N, Fageiry SK, Jenkins M, Garratt AN, Birchmeier C, Bennett DL. Axonally derived neuregulin-1 is required for remyelination and regeneration after nerve injury in adulthood. J Neurosci. 2011 Mar;31(9):3225-33. PMID: 21368034.

18. Wang G, Dai D, Chen X, Yuan L, Zhang A, Lu Y, Zhang P. Upregulation of neuregulin-1 reverses signs of neuropathic pain in rats. Int J Clin Exp Pathol. 2014 Aug;7(9):591621. PMID: 25337235.

19. Yildiz M, Karlidag T, Yalcin S, Ozogul C, Keles E, Alpay HC, Yanilmaz M. Efficacy of glial growth factor and nerve growth factor on the recovery of traumatic facial paralysis. Eur Arch Otorhinolaryngol. 2011 Aug;268(8):1127-33. PMID: 21259064.

20. Joung I, Yoo M, Woo JH, Chang CY, Heo H, Kwon YK. Secretion of EGF-like domain of heregulinbeta promotes axonal growth and functional recovery of injured sciatic nerve. Mol Cells. 2010 Nov;30(5):477-84. PMID:
20957456.

21. Fatani AJ, Al-Rejaie SS, Abuohashish HM, Al-Assaf A, Parmar MY, Ola MS, Ahmed MM. Neuroprotective effects of Gymnema sylvestre on streptozotocin-induced diabetic neuropathy in rats. Exp Ther Med. 2015 May;9(5):1670-8. PMID: 26136876.

22. Freeman RS, Burch RL, Crowder RJ, Lomb DJ, Schoell MC, Straub JA, Xie L. NGF deprivation-induced gene expression: after ten years, where do we stand? Prog Brain Res. 2004;146:111-26. PMID: 14699960.

23. Hellweg R, Hartung HD. Endogenous levels of nerve growth factor (NGF) are altered in experimental diabetes mellitus: a possible role for NGF in the pathogenesis of diabetic neuropathy. J Neurosci Res. 1990 Jun;26(2):258-67. PMID: 2142224.

24. Perlin JR, Lush ME, Stephens WZ, Piotrowski T, Talbot WS. Neuronal Neuregulin 1 type III directs Schwann cell migration. Development. 2011Nov;138(21):4639-48. PMID: 21965611.

25. Suzuki T, Sekido H, Kato N, Nakayama $Y$, Yabe-Nishimura C. Neurotrophin-3induced production of nerve growth factor is suppressed in Schwann cells exposed to high glucose: involvement of the polyol pathway. J Neurochem. 2004 Dec;91(6):1430-8. PMID: 15584919.

26.Jung WW, Kim HS, Shon JR, Lee M, Lee SH, Sul D, Na HS, Kim JH, Kim BJ. Intervertebral disc degeneration-induced expression of pain-related molecules: glial cell-derived neurotropic factor as a key factor. J Neurosurg Anesthesiol. 20110ct;23(4):32934. PMID: 21659885.

27. Li Y, Zhang Y, Liu DB, Liu HY, Hou WG, Dong YS. Curcumin attenuates diabetic neuropathic pain by downregulating TNF-alpha in a rat model. Int J Med Sci. 2013;10(4):377-81. PMID: 23471081.

28. Shi $X$, Chen $Y$, Nadeem L, Xu G. Beneficial effect of TNF-alpha inhibition on diabetic peripheral neuropathy. J Neuroinflammation. 2013 Jun;10:69. PMID: 23735240.

29. Marballi K, Quinones MP, Jimenez F, Escamilla MA, Raventos $H$, Soto-Bernardini 
MC, Ahuja SS, Walss-Bass C. In vivo and in vitro genetic evidence of involvement of neuregulin 1 in immune system dysregulation. J Mol Med (Berl). 2010 Nov;88(11):1133-41. PMID: 20625696.

\section{- Acknowledgements}

To Renmin Hospital and Central Laboratory of Renmin Hospital, Wuhan University, for assistance of the animal center.

\section{Correspondence:}

Zhongyuan Xia

Department of Anesthesia, Renmin Hospital of Wuhan University 238\# Jie Fang road Wu Chang District, Wuhan 430060

Hubei Province, China

Phone: +86 027-88041911-81024

xiazhongyuan2005@aliyun.com

Conflict of interest: none

Financial sources: none
Received: Sep 19, 2016

Review: Nov 18, 2016

Accepted: Dez 20, 2016
${ }^{1}$ Research performed at Central Laboratory, Remin Hospital, Wuhan University, Hubei, China. 